\title{
Design and Evaluation of a Telemonitoring Concept Based on NFC-Enabled Mobile Phones and Sensor Devices
}

\author{
Jürgen Morak, Hannes Kumpusch, Dieter Hayn, Robert Modre-Osprian, and Günter Schreier, Member, IEEE
}

\begin{abstract}
Utilization of information and communication technologies such as mobile phones and wireless sensor networks becomes more and more common in the field of telemonitoring for chronic diseases. Providing elderly people with a mobile-phonebased patient terminal requires a barrier-free design of the overall user interface including the setup of wireless communication links to sensor devices. To easily manage the connection between a mobile phone and wireless sensor devices, a concept based on the combination of Bluetooth and near-field communication technology has been developed. It allows us initiating communication between two devices just by bringing them close together for a few seconds without manually configuring the communication link. This concept has been piloted with a sensor device and evaluated in terms of usability and feasibility. Results indicate that this solution has the potential to simplify the handling of wireless sensor networks for people with limited technical skills.
\end{abstract}

Index Terms-Bluetooth, ECG, eHealth, near-field communication (NFC), telemonitoring.

\section{INTRODUCTION}

I NFORMATION and communication technologies (ICT) are used in telemonitoring applications to establish a virtual link between patients and their caregivers to support timely delivery of health-related data [1]. Utilization of ICT requires a barrierfree design of the overall patient terminal. Usability and Plugand-Play interoperability determines whether the patient is able to accept the system and willing to use it in daily routine.

In the following section, the utilization of mobile phones and wireless communication technologies as body and personal area network (PAN) will be discussed to introduce an intuitive and touch-based patient terminal.

\section{A. Mobile Phone as an Application Hosting Device}

The mobile phone evolved rapidly from a simple voice telephony device to a multifunction consumer electronics device incorporating personal information management functions, Web browsing, entertainment, and other forms of personal comput-

Manuscript received October 1, 2010; revised April 19, 2011 and October 13, 2011; accepted November 6, 2011. Date of publication November 18, 2011; date of current version February 3, 2012. This work was supported in part by the Styrian government, Department 3, science and research (Forschung Steiermark-Planung, Steuerung, Impulse-A3-22.E-4/2008-12).

The authors are with the Austrian Institute of Technology GmbH, 8020 Graz, Austria (e-mail: juergen.morak@ait.ac.at; hannes.kumpusch@ait.ac.at; dieter.hayn@ait.ac.at; Robert.modre@ait.ac.at; guenter.schreier@ait.ac.at). ing [2]. Thus, it is predestinated to serve as the patient's terminal device hosting an application to communicate with a remote telemonitoring service.

The hosted application manages data acquisition by interrogating sensor devices, processes and synchronizes those data, and receives and visualizes motivational and therapeutic feedback information [3].

Over the last decades, mobile network technologies advanced from Global System for Mobile communications to $4 \mathrm{G}$ networks like long-term evolution and worldwide interoperability for microwave access [4]. This evolution solved the problems regarding the connection to the telemonitoring center by providing high bandwidth and the possibility of being all-time online [1].

But the interplay between the mobile phone acting as an application hosting device (AHD) and medical sensor devices still remains an open issue. The majority of mobile phones on the market today are sophisticated devices with relatively complicated user interfaces. Mastery of their functions can sometimes take a long time [5].

\section{B. Personal/Body Area Networks}

By means of wireless PAN and body area network (BAN), the mobile phone serves as the hub of a set of portable or wearable sensors that monitor health-related parameters such as blood pressure, body weight, glucose, oxygen saturation, or even ECG signals [6]-[8].

1) Bluetooth: Bluetooth is the most popular PAN technology available in mobile phones. It is an industry standard for the wireless connection of portable devices and offers an operating range of up to $100 \mathrm{~m}$ at a theoretical data rate of up to $24 \mathrm{Mb} / \mathrm{s}$ (version 3.0 + HS) [9]. Bluetooth is an established and acknowledged technology in the medical environment [10] and has been selected by the Continua Health Alliance as PAN/BAN interface between the AHD and sensor devices [11]. For this purpose, the Bluetooth special interest group specified the Bluetooth health device profile (HDP) [12] and presented Bluetooth low energy (LE) as a feature of Bluetooth v4.0 [13] to provide fast system setup and LE communication for vital monitoring applications.

But so far, handling Bluetooth-enabled devices and making them communicate is not intuitive due to the setup procedure. Using mobile phones, this procedure includes several manual steps to perform the device/service discovery and pairing process [14]. Handling this complex configuration procedure (device/service discovery and pairing) may overstrain especially elderly patients [15]. 
To improve the usability during the pairing procedure as well as the protection against passive eavesdropping and man-inthe-middle (MITM) attacks the simple secure pairing (SSP) mechanism has been added to the Bluetooth specification v2.1+ EDR [16] and its successor versions. One method of SSP called Out of Band (OOB) uses an additional carrier to automatically exchange the pairing information. In [17], a concept is presented to bypass the Bluetooth device/service discovery by using radio frequency identification (RFID) tags. Similar approaches are pursued by the connection handover specification from the nearfield communication (NFC) Forum [18].

2) NFC: NFC is a wireless connectivity technology evolving from a combination of contactless identification and common networking technologies. Physical link is based on magnetic inductive coupling as used in other RFID standards like ISO14443. Besides peer-to-peer communication between two devices, an NFC device can switch between reader/writer mode as well as tag-emulation mode. When integrated into a mobile phone with mobile data services, these three modes allow for a broad range of new applications [19]. In the recent past, a number of trials and research projects have been conducted focusing on the usage of NFC in ticketing and payment applications as well as social networking and location-based services [20]-[22].

The short communication range is the major feature of this technology, since communication between two NFC enabled devices occurs when they are brought within a few centimeters to each other. This physical proximity of the device to the reader gives users the reassurance of being in control of the process. These properties render NFC suitable to act as PAN/BAN interface in the field of mHealth [23]. Due to NFCs broad application area even in medical applications, the NFC forum initiated the development of a technical specification for personal health device communications based on NFC [18].

In [24], a telemonitoring system is outlined that employs NFC technology for the entire patient equipment. It utilizes NFCenabled mobile phones as AHD, ID cards based on contactless smartcards to identify patients, and medical sensor devices to be interrogated by the mobile phone. For this reason, medical sensor devices like blood pressure meter and body weight scales have been enabled with NFC technology to provide the recent measured value set. The entire procedure of data acquisition can be performed without any key press. A Java 2 Micro Edition (J2ME) application installed on the mobile phone launches automatically by simply touching the ID card and guides the patient through the touch-based data acquisition process. Besides, interrogating medical sensor devices users are touching passive RFID tags with imprinted icons to acquire nonquantitative data like wellbeing or to answer questions presented on the phone's display.

One problem that this NFC-based system faced so far was the acquisition of a huge amount of data either in case of realtime transmission (i.e., ECG stream) or reading out a device's memory. Hundreds of kilobytes or even megabytes may take quite long if transmitted via NFC and would oblige the user to steadily keep the mobile phone close to the NFC-enabled sensor device as long as data are transmitted. In order to integrate a realtime streaming ECG sensor to the existing system, the usage of a communication protocol with wider range such as Bluetooth
TABLE I

COMPARISON OF BLUETOOTH AND NFC

\begin{tabular}{lll}
\hline \hline \multicolumn{1}{c}{ properties } & \multicolumn{1}{c}{ Bluetooth } & \multicolumn{1}{c}{ NFC } \\
\hline \hline frequenzy & $\begin{array}{l}2,4 \mathrm{GHz} \text { and 5 GHz } \\
\text { (Version 3.0 + HS) }\end{array}$ & $13,56 \mathrm{MHz}$ \\
\hline data rate & $\begin{array}{l}24 \mathrm{MBit} / \mathrm{s} \\
\text { (Version 3.0 }+\mathrm{HS})\end{array}$ & $424 \mathrm{kBit} / \mathrm{s}$ \\
\hline range & $100 \mathrm{~m}$ (class1) & $<10 \mathrm{~cm}$ \\
\hline set-up time & $>5 \mathrm{~s}$ & $<0,1 \mathrm{~s}$ \\
\hline $\begin{array}{l}\text { configuration/ } \\
\text { selectivity }\end{array}$ & manually & automatically \\
\hline topologie & $\begin{array}{l}\text { net (up to 255 } \\
\text { participants) }\end{array}$ & $\begin{array}{l}\text { peer 2 peer } \\
\text { (only 2 participants) }\end{array}$ \\
\hline compatibel to RFID & no & ISO14443, ISO15693 \\
\hline usability & data centric & $\begin{array}{l}\text { human centric, easy, } \\
\text { intuitive, fast }\end{array}$ \\
\hline
\end{tabular}

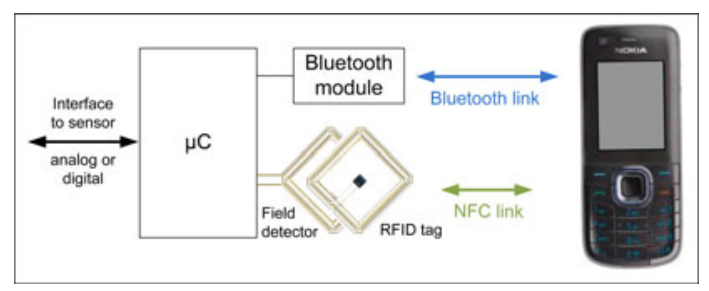

Fig. 1. Components to establish a Bluetooth connection with a mobile phone by bringing devices close together. The communication module consists of a $\mu \mathrm{C}$, a Bluetooth module, an RFID tag, and a field detector.

TABLE II

DATA STORED ON RFID TAG

\begin{tabular}{ll}
\hline \hline parameter & \multicolumn{1}{c}{ value } \\
\hline \hline MAC & 0018DA000D60 \\
\hline service name & COM1 \\
\hline
\end{tabular}

is unavoidable but derogates the usability of the given solution due to the need of the manual Bluetooth configuration. Table I compares the properties of Bluetooth and NFC.

3) Goal of This Study: The aim of this paper is to develop a concept that solves the problem of integrating a medical sensor device providing a huge amount of data to an existing telemonitoring system based on currently available NFC handsets. Touch-based intuitive data acquisition should be enabled even for live data streams and arbitrary amounts of data.

\section{METHODS}

\section{A. Technical Concept}

The intended concept was based on a combination of Bluetooth and NFC technology where NFC was used to activate Bluetooth and to exchange the information needed to establish a Bluetooth link. The first part of this setup was given by a mobile phone that provided both Bluetooth and NFC capability. The following core components have been selected to realize the second part for NFC supported Bluetooth communication (see Fig. 1).

1) Bluetooth module to communicate with the mobile phone via serial port profile (SPP) in transparent mode;

2) RFID tag to store essential data (see Table II) used for establishing a Bluetooth connecting

3) field detector to trigger the activation of Bluetooth;

4) microcontroller $(\mu \mathrm{C})$ to host the stack that manages the Bluetooth communication and to execute the main application gathering the data from analog and digital inputs. 


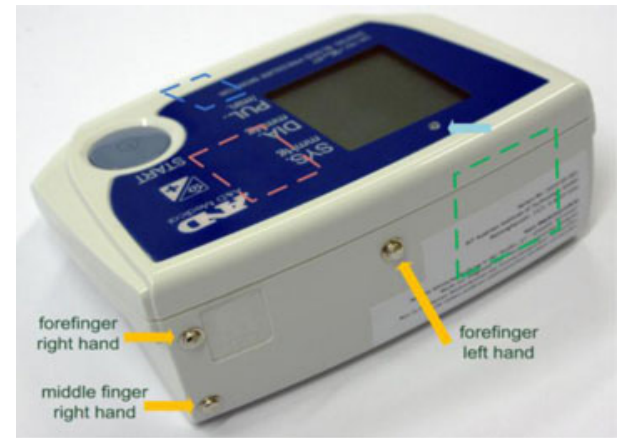

Fig. 2. Modified blood pressure meter with three electrodes (orange arrows), integrated $\mu \mathrm{C}$ with ECG amplifier (green square), Bluetooth module (blue square), RFID tag with field detector (red square), and light-emitting diode (light blue arrow). The electrodes have to be touched with the fingertips of the left and right forefinger as well as the right middle finger (reference electrode).

\section{B. Prototype Implementation}

The developed hardware platform for NFC-supported Bluetooth communication has been utilized to realize a prototype for the combined acquisition of blood pressure and an ECG. Therefore, it has been extended with an analog ECG front end and integrated into the off-the-shelf available blood pressure meter UA-767 (A\&D, Tokyo, Japan) (see Fig. 2).

The microcontroller MSP430F2410 (Texas Instruments, Dallas, TX) was chosen to act as the system's application processor. This type of chip offered two independent universal serial receive transmit (UART) interfaces and a couple of analog input channels.

The ECG part of the system was based on an analog circuit consisting of the AD620 (Analog Devices, Norwood, MA) and a second-order bandpass $(0.2-125 \mathrm{~Hz})$ to filter the signal. A driven right leg circuit was used to reduce common-mode interference. The analog signal was amplified by 1400 and connected to one of the $\mu \mathrm{C}$ 's analog input pins. Thus, the $\mu \mathrm{C}$ recorded a singlechannel ECG signal (e.g., Einthoven I) with a resolution of 8 bit at a sampling frequency of $250 \mathrm{~Hz}$. All components have been fitted inside the blood pressure meter's enclosure and connected to three metallic dry electrodes fixed on the meter's rear side (see Fig. 2).

One of the $\mu \mathrm{C}$ 's UART interfaces was connected to the blood pressure meter's serial interface. The $\mu \mathrm{C}$ was able to start a measurement and automatically received a few bytes of data (systole, diastole, heart rate) after measurement has finished. The second UART interface and further digital I/O lines were linked to a Bluetooth module type BNC4 (Amber Wireless, Cologne, Germany) which has been embedded separately into the meter's enclosure.

An RFID tag type Mifare Classic (NXP Semiconductors, Eindhoven, The Netherlands) with $1 \mathrm{~kb}$ of memory was programmed with the information of the respective Bluetooth module. This tag had a size of $45 \times 45 \mathrm{~mm}$ and was stuck behind the cover plate right next to the display window.

The antenna for the field detector has been realized by a flat coil with eight turns of copper that was attached to the inner side of the meter's cover plate congruent with the RFID tag.

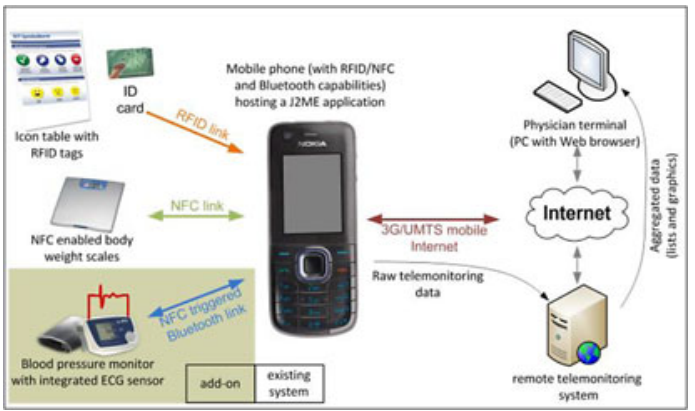

Fig. 3. Overview of the existing telemonitoring system (white area) based on an NFC/RFID and Bluetooth enabled mobile phone (Nokia 6212, Nokia, Espoo, Finland) acting as an AHD. The developed prototype (green area) was added to the existing system by means of adapting the J2ME application to establish the NFC triggered Bluetooth link. Data were collected from several active devices and passive items using RFID, NFC, and Bluetooth technology and sent to the remote telemonitoring system using secure https connection based on 3G/UMTS network.

\section{Integration Into the Mobile Telemonitoring System}

The developed prototype for the concurrent acquisition of blood pressure and ECG with NFC triggered Bluetooth connection has been integrated into an existing telemonitoring system (see Fig. 3). The following already existing NFC components have been used.

1) ID Card:: A contactless Smartcard type Mifare Classic was utilized as security token to identify the patient and authenticate access to the telemonitoring system. Touching this ID card with the mobile phone resulted in an automatic launch of the installed J2ME application.

2) Body Weight Scales: The body weight scales type UC321 PL (A\&D, Tokyo, Japan) has been fitted with NFC capabilities.

3) Smart Icon Table: An A4 sized table has been printed with several $50 \times 50 \mathrm{~mm}$ sized icons. Behind each icon, an RFID tag was stuck that electronically stored the meaning of the respective icon. These icons were intended to be touched with the mobile phone to answer questions about well-being and medication intake (shown on the display).

To include ECG recording using the developed prototype device, the J2ME application running on the mobile phone had to be adapted. When touching the ID card, the preinstalled application launches automatically and logs in at the remote telemonitoring system using the credentials lodged on this ID card. All data acquired from now will be uploaded to the patient record associated with this ID card. The user interface guided the patient through the data acquisition workflow, using a visual and acoustic interface - telling the patient, which step she/he had to take next. During ECG recording, the signal was streamed to the mobile phone and displayed in real time.

To upload the acquired data, the J2ME application running on the mobile phone was linked to a remote telemonitoring system using secured https communication based on 3G/UMTS mobile Internet services. Data were stored in the database of the remote telemonitoring system from where they could be accessed by physicians using a Web browser. After secure login, the user interface allowed us to view measurement lists, charts, and ECG recordings using an interactive viewer. 


\section{Evaluation}

The developed prototype device has been evaluated in terms of performance and feasibility.

1) Performance Test: This test dealt with the performance of the communication between sensor device and mobile phone. The Nokia 6131 NFC and the Nokia 6212 classic (Nokia, Espoo, Finland) were used to perform ten cycles of establishing the Bluetooth connection. Each handset was tested with different alignments to read the RFID tag. In this test, the J2ME application was already running. For each attempt to establish a Bluetooth connection, the incidence of both required events was documented: reading the RFID tag and triggering of the field detector.

2) Cross-Over Test: To evaluate Plug-and-Play interoperability, five units of the developed prototype as well as five different mobile phones were used to perform data acquisition. In the first part, one mobile phone type Nokia 6212 Classic connected to the five devices one after the other and controlled them to record ECG signals. In the second part of this test, three Nokia 6212 Classic and two Nokia 6131 NFC were used consecutively to control a single device for recording ECG signals.

3) Feasibility Test: The system's feasibility was evaluated in a test scenario where all parameters had to be acquired. Test persons were asked to use all components of the patient terminal according the following workflow.

1) Launch the J2ME application by touching the ID card.

2) Touch the blood pressure meter with the mobile phone.

3) Put on the cuff and touch the electrodes with the fingertips until the measurement has been finished.

4) Scale the body weight and touch the attached NFC module with the mobile phone.

5) Touch one of the three icons to indicate about wellbeing.

6) Touch "yes," "no," "more," or "less" to document the intake of medication number one.

7) Repeat the last step for medication number two.

\section{RESULTS}

This section consists of the workflow of the designed concept to establish a connection, the workflow of the resulting patient terminal, and the outcome of the system's evaluation.

\section{A. Workflow}

The designed concept provided the following workflow to establish a Bluetooth connection between a mobile phone and the developed communication module (see Fig. 4).

Both components were in idle mode. The mobile phone had Bluetooth and NFC switched on permanently and scanned its environment for RFID tags. The communication module was in sleep mode with Bluetooth being deactivated.

By means of bringing those devices close together, the mobile phone recognized the RFID tag. This leads to an automatic launch of the installed J2ME application which was provided with the data read from the RFID tag. Simultaneously, the field detector recognized the field generated by the mobile phone and triggered the $\mu \mathrm{C}$ to wake up from sleep mode. In the following, the $\mu \mathrm{C}$ enabled the Bluetooth chip and configured it to be addressed as slave and to operate the SPP.

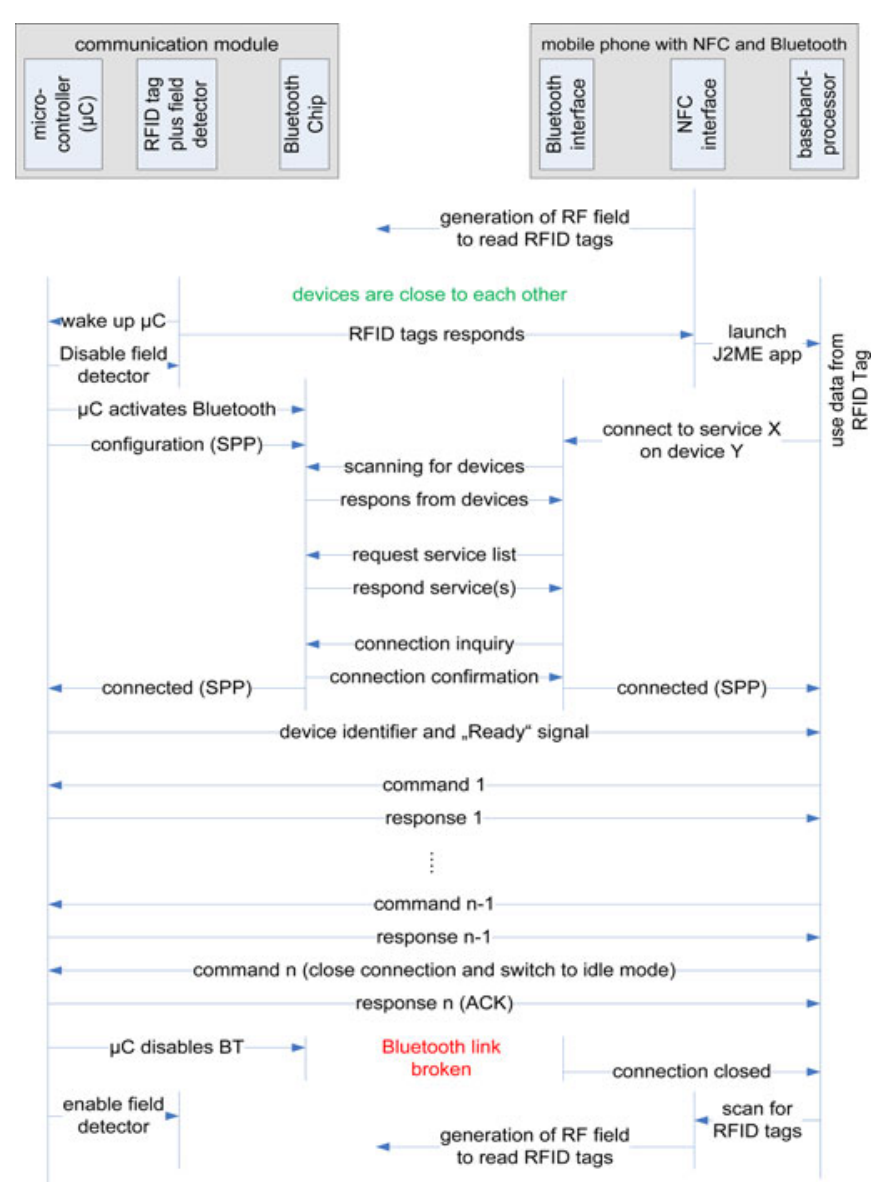

Fig. 4. Cycle of establishing a Bluetooth connection triggered and configured by NFC.

The J2ME application requested the mobile phone's Bluetooth module to establish a connection to a dedicated service (X) on a dedicated device (Y) defined by the MAC addresses. After the service was found, an inquiry to establish a connection was sent. Once the module's Bluetooth chip received the connection inquiry, it permitted to establish this connection and indicated the $\mu \mathrm{C}$ that there was a connection. The $\mu \mathrm{C}$ then sent a string back to the Bluetooth module that was transparently transmitted to the mobile phone indicating the device identifier and the status of being ready to receive commands. Thus, the mobile phone was able to communicate with the $\mu \mathrm{C}$ in a command-response manner.

After all data were exchanged, the mobile phone sent the final command to the $\mu \mathrm{C}$ to close the connection by disabling the Bluetooth chip and to jump back to sleep mode. The $\mu \mathrm{C}$ acknowledged and subsequently performed this command. It remained in this state until it was triggered by the field detector to wake up again. The application recognized the closed Bluetooth connection and terminated automatically after processing the received data.

\section{B. Prototype Integration in Medical Application}

The developed communication module was then integrated into a blood pressure meter and combined with an ECG recording component to evaluate the designed concept as part of a medical application. 
For data acquisition, the user had to touch the ID card that launched the J2ME application automatically and authenticated the user. A voice sounded and asked the user to bring the mobile phone close to the extended blood pressure meter right next to the meter's display. This action triggered the Bluetooth setup process. At the same time, the user was asked to put on the cuff of the blood pressure meter and to touch the ECG electrodes with her/his fingertips.

After $10 \mathrm{~s}$, the blood pressure measurement and ECG recording started automatically. ECG data were transmitted to the mobile phone in real time to be displayed. As soon as the blood pressure measurement had finished, ECG streaming was stopped. If the blood pressure meter was not able to provide a valid measurement, the ECG recording stopped automatically after $90 \mathrm{~s}$. Finally, the user had to acquire further health parameters by touching the NFC-enabled body weight scales and the icon table. Once all information was acquired, the application transmitted the data and terminated automatically.

\section{Evaluation}

The following results have been obtained from the performed tests described earlier.

1) Performance Test: This test investigated the interplay of the mobile phone with the RFID tag and the field detector. The Nokia 6131 NFC, a clamshell phone, and the Nokia 6212 Classic, a bar type phone, were used to touch the meter's enclosure at the position where the RFID tag/field detector was located. They were aligned in four different directions (vertical or horizontal with display up or down) to read the RFID tag. Ten attempts per direction and mobile phone have been performed. The results obtained from this test indicate that the Nokia 6131 NFC has a better reading performance. Independent from the mobile phone's direction, all attempts to read the RFID tag as well as all attempts to trigger the field detector were successful. This resulted in $100 \%$ of established Bluetooth connections.

Using the Nokia 6212 Classic showed different results depending on the direction. Holding the mobile phone vertically with display to front only four attempts resulted in successful Bluetooth connections of four successful read-outs of the RFID tag. A horizontal direction with display to front revealed similar results of only three successful connections. Holding this mobile phone with its display down onto the device also resulted in $100 \%$ of successful connection independent whether it was aligned horizontal or vertical.

2) Cross-Over Test: This test revealed that each attempt to establish a connection either in part one ore part two of this test was successful without the need of a manual interaction using the devices keypad.

In case the field detector recognized any NFC enabled mobile phone which did not attempt to establish a connection to the sensor device, it automatically switched OFF Bluetooth after 20 $\mathrm{s}$ and went to idle mode again.

3) Feasibility Test: To evaluate the feasibility of the overall system, 12 healthy volunteers (three female, mean age 34 . $5 \pm 8.3$ years) performed the same data acquisition procedure at least five times on a single day. They used the Nokia 6212
Classic and always touched the RFID tag of the blood pressure meter with the phone's display down. Each attempt to establish the connection between mobile phone and the device was successful. A total of 503 data objects were acquired and uploaded in 63 successful transmissions. A single case occurred where no blood pressure value was measured but only a 90-s ECG record.

\section{Discussion}

The presented concept provides an intuitive solution for establishing a wireless link between a mobile phone and a medical sensor device. The system is based on standard communication technologies and allows for transmitting data over a distance of several meters at a transfer rate of more than $1 \mathrm{Mb} / \mathrm{s}$. By means of bringing an NFC-enabled mobile phone close to the medical sensor device, the Bluetooth connection gets established automatically without any prior configuration. This link can be used for real-time streaming of biosignals as well as to read out an arbitrary amount of data. In addition to that, it can even be used to configure and control the device which provides the option to develop sensor devices without any button or display. The complete user interface can be shifted to the mobile phone.

In terms of functionality, the developed prototype is comparable to the concept proposed in [17] and the static connection handover procedure specified by the NFC Forum [18]. RFID tags are used to provide the mobile phone with the information required to bypass the Bluetooth device/service discovery procedure. While these two concepts would require Bluetooth to be activated permanently on the sensor device, our concept utilizes a field detector to recognize the intention to establish a link. Thus, it automatically activates the system and enables Bluetooth which provides a valuable improvement in terms of power management. The OOB method [16] and the negotiated handover procedure [18] do also provide Bluetooth activation initiated by bringing the devices close together but require an NFC chip on the sensor device side.

OOB and connection handover methods were designed primarily to setup a permanently paired Bluetooth link which reconnects automatically when devices are switched ON or brought within a certain range (as also demonstrated in [25]). Opposite to this, our approach aims at establishing a new communication link on demand, similar to pure NFC.

In terms of security, $\mathrm{OOB}$ and negotiated handover procedure are more secure due to mutual authentication which so far provides best protection against MITM attacks [14] but depends on the level of MITM protection of the NFC link. RFID tags store static data and can even be hacked and manipulated [26], which make the pairing process vulnerable if not protected by additional measures like signatures [27].

Similar to the system demonstrated in [17], the presented prototype has not been secured against eavesdropping and MITM attacks. This situation is closely related to the types of mobile phones (Nokia 6131 and 6212) that had to be used at the time the prototype was developed, since they were the first and the only commercially available handsets with integrated NFC capabilities. Both phones are running Nokia's proprietary S40 firmware which is able to execute third party J2ME applications. On these 
TABLE III

LINKING TECHNOLOGIES, DATA, AND USE CASES

\begin{tabular}{lll}
\hline \hline \multicolumn{1}{c}{ link } & \multicolumn{1}{c}{ data } & \multicolumn{1}{c}{ use case } \\
\hline \hline RFID & $\begin{array}{l}\text { few bits or bytes } \\
\text { (static) }\end{array}$ & $\begin{array}{l}\text { identification, well being, } \\
\text { medication intake, activity }\end{array}$ \\
\hline NFC & $\begin{array}{l}\text { bytes to kbytes } \\
\text { (dynamic) }\end{array}$ & $\begin{array}{l}\text { blood pressure, body weight, } \\
\text { blood sugar, } \mathrm{O}_{2} \text { saturation, } \\
\text { short term biosignals }\end{array}$ \\
\hline Bluetooth+NFC & $\begin{array}{l}\text { kbytes to Mbytes } \\
\text { (dynamic) }\end{array}$ & $\begin{array}{l}\text { streamed biosignals, ECG data, } \\
\text { 3D accelerometer data, } \\
\text { plethysmography }\end{array}$ \\
\hline
\end{tabular}

devices, this is the only way to combine the mobile phone's NFC and Bluetooth features. Unfortunately, using the J2ME framework, it is neither possible to enable Bluetooth within the application nor to set a PIN code automatically when establishing an authenticated and encrypted Bluetooth connection [28]. Thus, we decided to operate Bluetooth without a PIN so as to avoid that the user has to enter the PIN code manually after touching the device. As a consequence, the prototype did not provide an encrypted link between the sensor device and the mobile phone. Additionally, Bluetooth has to be activated permanently which has a negative impact on the longevity of the battery (NFC also needs some battery power but since NFC gets deactivated automatically when the mobile phone is in stand-by mode, it needs much less energy as compared to Bluetooth).

It can be expected that in future designs, these problems will be solved by employing mobile handsets providing an improved NFC-Bluetooth interplay. A promising way would be to combine NFC and Bluetooth on a hardware level as presented in [29] or at least on the mobile phone's operating system level. An NFC-enabled smartphone running an operating system like Symbian (Nokia, Espoo, Finland) or Android (Google Inc., Mountain View, CA) could execute an application with native hardware access and solve both issues mentioned earlier.

Since the proposed solution establishes a new link each time the sensor device is touched, it can be integrated seamlessly into the existing NFC-based telemonitoring system. Hence, our approach allows for acquiring all kind of health parameters in the same way-simply by touching items or devices. Table III gives an overview of use cases covered by this concept.

This Plug-and-Play concept without having Bluetooth paired permanently provides two major advantages. The minimized configuration effort of this approach allows for easy replacement in case of a broken device. Just shipping a new device by mail without the need of any configuration or installation procedure would disburden patients, caretaking staff, and relatives. Additionally, the fact of keeping the devices unpaired allows for a multiuser scenario where devices can be shared among several users living in the same place-each having their own mobile phone. Those two advantages come at the prize of additional costs for the NFC infrastructure which are expected to decrease in the medium term due to the predicted market share of future NFC-enabled handsets.

The multiuser scenario is realized by using the ID card to identify and authenticate the patient. When touching the ID card, the preinstalled application launches automatically and connects to the remote telemonitoring system using the credentials stored on this ID card. All subsequently acquired data will be uploaded to the electronic patient record associated with this ID card. Thus, the patient has to take care of his/her ID card and is requested to contact the helpdesk in case of loss. From a security point of view, it is possible to read the data stored on the body weight scale with any other mobile phone running an appropriate NFC reader application. However, reading out just the single stored last measurement value from most of such measuring devices is always possible via the display and as such no security risk. To avoid, however, that a value would be uploaded twice-either by the same or another user-we implemented a filter mechanism based on the device's serial number and a unique session identifier for each measured value. In case of the described prototype-where a blood pressure and ECG sensor has been combined-it would be quite complex to read out the data by an unauthorized person's mobile phone due to the fact that the data are streamed in real time and not stored to be read afterward. Spying out or manipulating these data would require an eavesdropping or MITM attack. This can be prevented in future designs by using an encrypted link based on the concept described earlier.

The developed prototype used the SPP and a proprietary data format. In future mHealth applications, a broad range of wireless technologies such as NFC, classic Bluetooth, as well as the upcoming Bluetooth LE version will be utilized. Since each of those provides its own HDP, interoperability concepts will have to be developed to allow for coexistence and interoperability across of all them.

We are aware of the fact that the presented usability results cover only basic aspects of the presented approach. Eventually, the usability has to be assessed in the target group of patients with heart disease of higher age and in the context of a telehealth setting. For this, we have planned an appropriate clinical trial together with a clinical partner and already got approval from the ethics committee of the Medical University of Graz.

\section{CONCLUSION}

A concept to easily establish a Bluetooth connection has been designed and implemented prototypically. By means of combining Bluetooth with NFC technology, an easy-to-establish wireless interface in between sensor devices and mobile phones was realized. Although the prototype shows some weaknesses in terms of security, it provides high usability due to the intuitive and seamless acquisition of all kind of monitoring data including biosignals just by touching icons and sensor devices with a mobile phone.

\section{REFERENCES}

[1] L. Al-hakim, Web and Mobile-Based Applications for Healthcare Management, Hershey, PA: IRM Press, 2007.

[2] P. A. Hedtke, "Can wireless technology enable new diabetes management tools?," J. Diabetes Sci. Technol., vol. 2, no. 1, pp. 127-130, Jan. 2008.

[3] G. Giménez-Pérez, M. Gallach, E. Acera, A. Prieto, O. Carro, E. Ortega, J. M. González-Clemente, and D. Mauricio, "Evaluation of accessibility and use of new communication technologies in patients with type 1 diabetes mellitus," J. Med. Internet Res., vol. 4, no. 3, p. E16, Dec. 2002.

[4] K. Patrick, W. G. Griswold, F. Raab, and S. S. Intille, "Health and the mobile phone," Amer. J. Prev. Med., vol. 35, no. 2, pp. 177-181, Aug. 2008.

[5] D. L. Katz and B. Nordwall, "Novel interactive cell-phone technology for health enhancement," J. Diabetes Sci. Technol., vol. 2, no. 1, pp. 147-153, Jan. 2008.

[6] T. Norgall, R. Schmidt, and T. Von Der Grun, "Body area network: A key infrastructure element for patient-centered telemedicine," in Wearable eHealth Systems for Personalised Health Management-State of the Art 
and Future Challenges, A. Lymberis and D. deRossi, Eds. Amsterdam, The Netherlands: IOS Press, 2004.

[7] A. A. Tahat, "Mobile messaging services-based personal electrocardiogram monitoring system," Int. J. Telemed. Appl., vol. 2009, p. 859232, 2009.

[8] M. N. Kamel Boulos, R. C. Lou, A. Anastasiou, C. D. Nugent, J. Alexandersson, G. Zimmermann, U. Cortes, and R. Casas, "Connectivity for healthcare and well-being management: Examples from six European projects," Int. J. Environ. Res. Public Health., vol. 6, no. 7, pp. 19471971, Jul. 2009.

[9] Bluetooth SIG (Sep. 2010). Datasheet of Bluetooth Version 3.0 + HS [Online]. Available: http://www.bluetooth.com/SiteCollectionDocuments/ HS_Doc_Web.pdf.

[10] A. H. More, "Bluetooth low energy: Wireless connectivity for medical monitoring," J. Diabetes Sci. Technol.., vol. 4, no. 2, pp. 457-463, Mar. 2010.

[11] R. Cnossen, B. Jorgensen, J. Krishna, C. McClellan, and R. Rogers, "Continua enables standards-based mobile personal health solutions," Telemed. J. E. Health., vol. 16, no. 4, pp. 393-399, May 2010.

[12] J. Noueihed, R. Diemer, S. Chakraborty, and S. Biala, "Comparing Bluetooth HDP and SPP for mobile health devices," in Proc. Int. Conf. Body Sensor Networks, 2010, pp. 222-227.

[13] Bluetooth SIG (Jun. 30, 2010). Bluetooth Specification Version 4.0 Low Energy (Retrieved April 15, 2011) [Online]. Available: https://www. bluetooth.org/docman/handlers/downloaddoc.ashx?doc_id = 229737 .

[14] D. Sharmila, R. Neelaveni, and K. Kiruba, "Bluetooth man-in-the-middle attack based on secure simple pairing using out of band association model," in Proc. Int. Conf. Control, Automat., Commun. Energy Conservation, Jun., 2009, pp. 1-6.

[15] R. S. Istepanian, K. Zitouni, D. Harry, N. Moutosammy, A. Sungoor, B. Tang, and K. A. Earle, "Evaluation of a mobile phone telemonitoring system for glycaemic control in patients with diabetes," J. Telemed. Telecare., vol. 15, no. 3, pp. 125-128, 2009.

[16] Bluetooth SIG (Jul. 26 2007). Bluetooth Specification Version 2.1 + EDR (Retrieved September 28, 2010) [Online]. Available: http:// www.bluetooth.com/Specification\%20Documents/Core_V21_EDR.zip.

[17] T. Salminen, S. Hosio, and J. Riekki, "Enhancing Bluetooth connectivity with RFID," in Proc. 4th Annu. IEEE Int. Conf. Pervasive Comput. Commun., Mar., 2006, pp. 36-41.

[18] NFC Forum. (Apr. 15 2011). Specification overview [Online]. Available: http://www.nfc-forum.org/specs/spec_dashboard/.

[19] J. Fischer, "NFC in cell phones: The new paradigm for an interactive world," IEEE Commun. Mag., vol. 47, no. 6, pp. 22-28, Jun. 2009.

[20] S. Miranda and N. Pastorelly, "NFC mobiquitous information service prototyping at the University of Nice Sophia Antipolis and multi-mode NFC application proposal," in Proc. 3rd Int. Workshop Near Field Commun., Feb., 2011, pp. 3-8.

[21] J. Neefs, F. Schrooyen, J. Doggen, and K. Renckens, "Paper ticketing versus electronic ticketing based on off-line system 'tapango'," in Proc. 2nd Int. Workshop Near Field Commun., Apr., 2010, pp. 3-8.

[22] J. Haikio, T. Tuikka, E. Siira, and V. Tormanen, "Would you be my friend?'-Creating a mobile friend network with 'hot in the city'," in Proc. 43rd Hawaii Int. Conf. Syst. Sci., Jan. 2010, pp. 1-10.

[23] E. Strömmer, J. Kaartinen, J. Pärkkä, A. Ylisaukko-Oja, and I. Korhonen, "Application of near field communication for health monitoring in daily life," in Proc. Conf. Proc. IEEE Eng. Med. Biol. Soc., 2006, vol. 1, pp. 3246-3249.

[24] P. Kastner, J. Morak, R. Modre, A. Kollmann, C. Ebner, F. M. Fruhwald, and G. Schreier, "Innovative telemonitoring system for cardiology: From science to routine operation," Appl. Clin. Informat., vol. 1, no. 2, pp. 165$176,2010$.

[25] S. Wagner, "Zero-configuration of pervasive healthcare sensor networks," in Proc. 3rd Int. Conf. Pervasive Comput. Appl., Oct., 2008, vol. 1, pp. 405408.

[26] F. D. Garcia, P. van Rossum, R. Verdult, and R. W. Schreur, "Wirelessly pickpocketing a mifare classic card," in Proc. 30th IEEE Symp. Security Privacy, May 2009, pp. 3-15.

[27] M. Roland and J. Langer, "Digital signature records for the NFC data exchange format," in Proc. 2nd Int. Workshop Near Field Commun., Apr., 2010, pp. 71-76.

[28] R. Verdult and F. Kooman, "Practical attacks on NFC enabled cell phones," in Proc. 3rd Int. Workshop Near Field Commun., Feb., 2011, pp. 77-82.

[29] Y. Anokwa, G. Borriello, T. Pering, and R. Want, "A user interaction model for NFC enabled applications," in Proc. 5th Annu. IEEE Int. Conf. Pervasive Comput. Commun. Workshops, Mar., 2007, pp. 357-361. 